doi: $10.13108 / 2016-8-4-42$

UDC 512.5

\title{
DARBOUX TRANSFORMATIONS IN THE INVERSE SCATTERING PROBLEM
}

\author{
M.SH. BADAKHOV, A.B. SHABAT
}

\begin{abstract}
As it is known, Darboux transformations play a key role in applications in ISP. In the paper we expose the theory of such transformations for the Schrödinger equations with compactly supported potential, which are not necessarily smooth. We study a new class of transformations connected with the zeroes of the reflection coefficient located at conjugated points in the complex plane.
\end{abstract}

Keywords: Inverse Problem, Schrödinger equation, compactly supported potentials, Darboux transformation.

Mathematics Subject Classification: 34L25, 35J10, 37K15

\section{Compactly Supported potentials}

The problem on enlarging the family of exactly solvable potentials in the inverse scattering problem for the Schrödinger equation attracts permanent attentions of the specialists. One of such classes of the potentials, for which the inverse scattering problem can be solved completely, is formed by Bargmann potentials (reflectionless potentials, see [6]). However, while solving the inverse problem in the class of the Bargmann potential, a character feature in the spectral data is missing. This feature is related with the threshold of the essential spectrum that, in particular, does not allow one to enlarge essentially this class by passing to the limit. Another class of the potentials admitting constructive solutions is formed by the delta-type potentials [2] and here we consider the enlarging of this class by means of special Darboux transforms preserving the property of compact supports for the potentials.

For the solutions to the Schrödinger equations

$$
L \psi=\lambda \psi, \quad L=q(x)-D_{x}^{2},
$$

the Darboux transform is introduced by the "well-known" formula

$$
\left(\begin{array}{c}
\hat{\psi} \\
\hat{\psi}_{x}
\end{array}\right)=\left(\begin{array}{cc}
f & -1 \\
-f^{2}+\lambda-\lambda_{0} & f
\end{array}\right)\left(\begin{array}{c}
\psi \\
\psi_{x}
\end{array}\right), \quad f_{x}+f^{2}=q(x)-\lambda_{0}
$$

by which we find that

$$
\begin{gathered}
\hat{\psi}=(f-D) \psi, \quad \hat{L} \hat{\psi}=\lambda \hat{\psi}, \quad \hat{L}=\hat{q}(x)-D^{2}, \quad \hat{q}(x)=q(x)-2 f_{x}, \\
\left(f+\frac{\hat{\psi}^{\prime}}{\hat{\psi}}\right)\left(f-\frac{\psi^{\prime}}{\psi}\right)=\lambda-\lambda_{0} .
\end{gathered}
$$

In these formulae, the choice of the function $f(x)$ satisfying the Riccati equation is determined by the property of being compactly supported for the original and transformed potentials.

M.Sh. Badakhov, A.B. Shabat, Darboux transformations in the inverse sCattering problem.

(C) Badakhov M.Sh., Shabat A.B. 2016.

The research is supported by the grant of the Russian Science Foundation (project no. 15-11-20007).

Submitted August 27, 2016. 
By the formulae (2) this means that the function $f(x)$ is constants both as $x \sim-\infty$ and as $x \sim+\infty$. As a rule, we can assume that

$$
\lambda_{0}=-k_{0}^{2}<0, \quad f(x)=\frac{\psi_{x}}{\psi}\left(x, k_{0}\right)=\left\{\begin{array}{rl}
k_{0}, & x \sim-\infty \\
-k_{0}, & x \sim \infty
\end{array} .\right.
$$

This corresponds to choosing the logarithmic derivative of the Jost function $\psi(x, k)$ as $f$; the Jost function is introduced as the solution to the integral equation

$$
\psi(x, k)=\phi(x, k) e^{k x}, \quad \phi(x, k)=1+\int_{-\infty}^{x} R(x-s, k) \phi(s, k) q(s) d s,
$$

with the kernel $R$. In the right half-plane $K_{+}$

$$
K_{+}=\{k \in \mathbb{C}, \operatorname{Re} k \geqslant 0\},
$$

this kernel satisfies the estimate

$$
R\left(x^{\prime}, k\right)=\frac{1-e^{-2 k x^{\prime}}}{2 k} \theta\left(x^{\prime}\right) \quad \Rightarrow \quad\left|R\left(x^{\prime}, k\right)\right| \leqslant \int_{-x^{\prime}}^{0}\left|e^{2 k s}\right| d s \leqslant x^{\prime}, \quad \operatorname{Re} k \geqslant 0 .
$$

To the left of the support of the potential, the Jost function is of the form $\psi(x, k)=e^{k x}$, while to the right it is given by

$$
\psi(x, k)=a(k) e^{k x}+b(k) e^{-k x}, \quad x \sim+\infty .
$$

The functions $a(k)$ and $b(k)$ have a first order pole at $k=0$ but their linear combination $a(k)+b(k)$ is regular at this point.

In the case we are interested in, the function $q$ is a linear combination of delta-functions. We call such functions delta-type. Choosing $\phi \equiv 1$ as the zero approximation and applying the iterations method to scalar integral equation (4), one can check that for the Schrödinger equation with a delta-type potential of the form

$$
q(x)=\sum_{j=1}^{N} \gamma_{j} \delta\left(x-x_{j}\right)
$$

the iterations method provides the exact solution at the $N$ th step. The constructed solution to Schrödinger equation (1) with potential (8) is continuous at all $x$ and satisfies the matching condition of left and right derivatives at all the nodes $x_{j}, j \in[N]$.

The case (3) corresponds to a special choice of the parameter $k_{0}>0$ associated with a negative eigenvalue $\lambda=\lambda_{0}<0$ of the original operator $L=q(x)-D^{2}$ and

$$
a\left(k_{0}\right)=0, \quad \psi\left(x, k_{0}\right) \rightarrow 0, \quad x \rightarrow \pm \infty .
$$

If the eigenvalue of $\lambda_{0}<0$ is minimal, then Jost function (9) has no zeroes and its logarithmic derivative has no poles. Employing (3), in this case for the transformed Jost function we find:

$$
\hat{\psi}(x, k)=\frac{f \psi-\psi_{x}}{k_{0}-k}=\hat{a}(k) e^{k x}+\hat{b}(k) e^{-k x}, \quad x \sim \infty ; \quad \hat{a}(k)=a(k) \frac{k+k_{0}}{k-k_{0}}, \quad \hat{b}(k)=-b(k) .
$$

We observe that in the case of the real-valued potentials $q(x), x \in \mathbb{R}$, on the vertical axis $k=i \xi, \xi \in \mathbb{R}$, the following "realness" conditions should be satisfied:

$$
a(-k)=\bar{a}(k), \quad b(-k)=\bar{b}(k), \quad|a(k)|^{2}=1+|b(k)|^{2}>0, \quad k=i \xi, \quad \xi \in \mathbb{R},
$$

where the bar stands for the complex conjugation. For arbitrary compactly supported potentials, the equation relating entire functions of exponential type $2 k a(k)$ and $2 k b(k)$ is written as

$$
a(k) a(-k)-b(k) b(-k)=1 .
$$


For even potential the equation is simplified and it can be rewritten as

$$
q(-x)=q(x) \Rightarrow b(-k)=-b(k), \quad a(k) a(-k)=(1-b(k))(1+b(k)) .
$$

While using the zeroes of the second coefficient $b\left(k_{1}\right)=0$, formula (2) gives:

$$
f(x)=\frac{\psi_{x}}{\psi}\left(x, k_{1}\right)=\left\{\begin{array}{ll}
k_{1}, & x \sim-\infty \\
k_{1}, & x \sim \infty
\end{array} \quad \Rightarrow \quad \tilde{a}(k)=a(k), \quad \tilde{b}(k)=b(k) \frac{k_{1}+k}{k_{1}-k} .\right.
$$

However, the roots of the function $b(k)$ are complex numbers and we need to make an additional transformation to the restore the realness of the potential. As a result of such double transformation we obtain

$$
\hat{a}(k)=a(k), \quad \hat{b}(k)=b(k) \frac{k_{1}+k}{k_{1}-k} \times \frac{k_{2}+k}{k_{2}-k}, \quad k_{2}=\bar{k}_{1} .
$$

The case, when all the zeroes of $b(k)$ are located at the vertical line, is of a special interest. In this case $k_{2}=-k_{1}$ and formula (14) leads us to an unexpected result:

$$
b(k)=0, \quad k=k_{1,2}= \pm i \xi, \xi \in \mathbb{R} \quad \Rightarrow \quad \hat{a}=a, \quad \hat{b}=b .
$$

We recall that in the case of "smooth" potentials the inverse scattering problem is reduced to a pair of integral equations

$$
\begin{aligned}
& \psi(x, k)=e^{k x}+\int_{-\infty}^{x} K(x, y) e^{k y} d y, \quad \operatorname{Re} k \geqslant 0, \\
& K(x, y)+F(x+y)+\int_{-\infty}^{x} K(x, s) F(s+y) d s=0, \quad y<x .
\end{aligned}
$$

By means of the Fourier transform of the Jost function (44), the first of them determines the kernel $K(x, y), y<x$, as a function of $y$ for a fixed $x$. The second of these relations, "GelfandLevitan-Marchenko" equation is used to recover the function $K(x, y), y<x$, as solutions to the Wiener-Hopf equation on the half-line $-\infty<y \leqslant x$ with a given kernel $F=F(x+y)$ tending to zero $X=x+y \rightarrow-\infty$. The Fourier transform of the function $F(X)$ is expressed in terms of $b / a$ on the vertical line $k=i \xi$. In Figure 1 , the graph of the absolute value for some function is provided, when all the zeroes of the entire function $2 k b(k)$ are located on the imaginary axis. As we shall show below, to construct the example of non-unique solvability of equation (17), one can use a pair of complex conjugate zeroes (15) of the entire function $2 k b(k)$ located at the imaginary axis. Observing that a pair of complex conjugate zeroes $k=i \xi$ corresponds to the same value $\lambda=-k^{2}$, we shall show (see Example 2 in the end of the work) that in this case the Darboux transform leads to a new potential with a support in the half-line $\left[x_{1}, \infty\right)$ but with the same function $F(x)$.

\section{ZEROES OF THE REFLECTION COEFFICIENT}

Under Darboux transformation (10), the coefficient $\hat{a}(k)$ has no zero at the point $k=k_{0}$ and hence, we can get rid of the discrete spectrum of Schrïodinger operator (1) in the inverse problem. We recall that the number of these eigenvalues coincides with the number of sign changes of the graph of the limiting (as $k \rightarrow 0$ ) Jost function $\psi(x, 0)$. In case (8) this graph is a polyline (see Figure 2) with the nodes at the points $x_{j}$ playing the main role in the spectral properties of delta-type potentials [1].

To give a complete picture, we recall the known formulae related with a piece-wise constant potential $q_{\varepsilon}$ with the shape of a rectangular well, which becomes the delta function with the coefficient $\gamma$ in the limit $\varepsilon \rightarrow 0$ :

$$
q(x)=q_{\varepsilon}(x)=\frac{\gamma}{\varepsilon}=-h, \quad x \in[-\varepsilon, 0] \stackrel{\text { def }}{=} I_{\varepsilon} .
$$




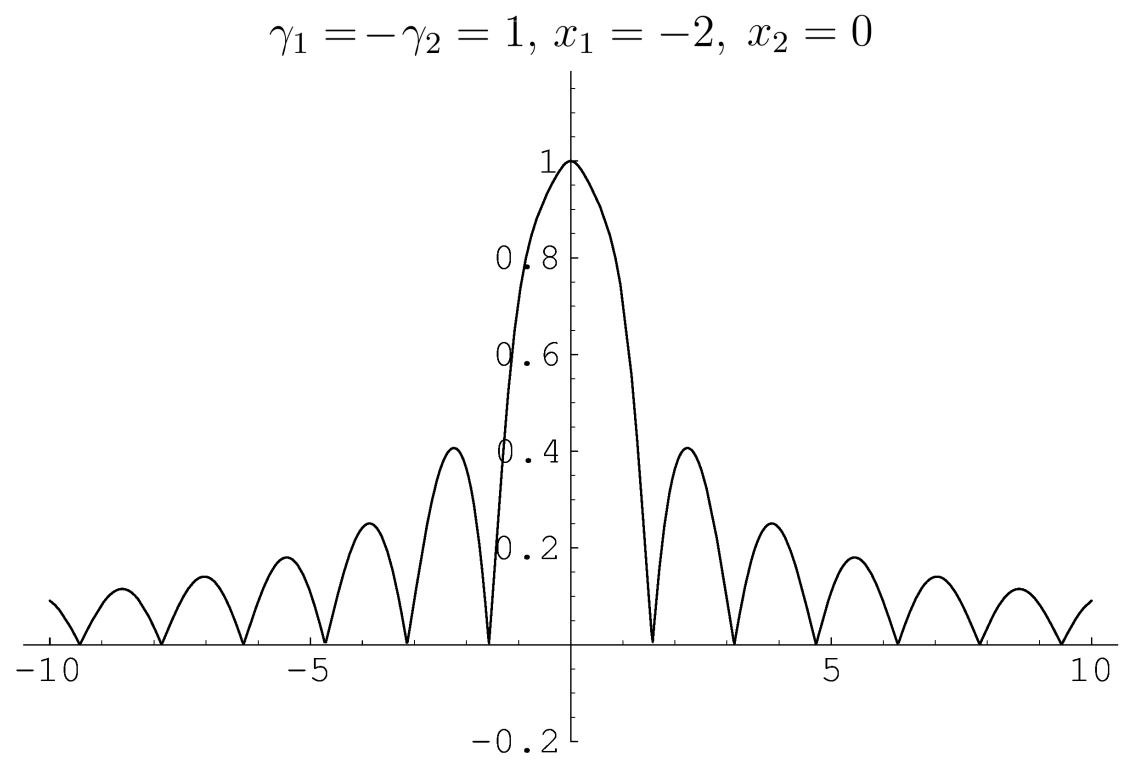

FIGURE 1. The graph of $\left|\frac{b}{a}\right|(k)$ on the real axis.

The Jost function $\psi_{\varepsilon}(x, k)$ and its derivative inside $I_{\varepsilon}$ are determined by the following formula:

$$
\begin{array}{ll}
e^{k \varepsilon} \psi(x, k)=\cosh z(x+\varepsilon)+\frac{k}{z} \sinh z(x+\varepsilon), & -\varepsilon \leqslant x \leqslant 0, \\
e^{k \varepsilon} \psi_{x}(x, k)=k \cosh z(x+\varepsilon)+z \sinh z(x+\varepsilon), & z^{2}=k^{2}-h .
\end{array}
$$

In particular, as $k=0(z=i \sqrt{h})$, we have

$$
\left.\psi\right|_{k=0}= \begin{cases}\cos (x+\varepsilon) \sqrt{h}, & -\varepsilon \leqslant x \leqslant 0 \\ \cos (\varepsilon \sqrt{h})-x \sqrt{h} \sin (\varepsilon \sqrt{h}), & x>0\end{cases}
$$

that, as it has been observed, allows us to determine the number of the eigenvalues depending on the quantity $\varepsilon$. On the other hand, these eigenvalues are determined by the zeroes in the right half plane (5) of the entire function $2 k a(k)$, where

$$
a(k) e^{k \varepsilon}=\cosh z \varepsilon+\frac{1}{2}\left(\frac{k}{z}+\frac{z}{k}\right) \sinh (z \varepsilon), \quad b(k) e^{k \varepsilon}=\frac{1}{2}\left(\frac{k}{z}-\frac{z}{k}\right) \sinh (z \varepsilon) .
$$

As an approximate solution $\phi$ to integral equation (44), we can use the first iteration

$$
\phi(x, k) \approx 1+\int_{-\infty}^{x} R\left(x-x^{\prime}, k\right) q\left(x^{\prime}\right) d x^{\prime}=\left\{\begin{array}{ll}
\frac{\gamma_{1}}{\varepsilon} \int_{-\varepsilon}^{x} R\left(x-x^{\prime}, k\right) d x^{\prime}, & x \in[-\varepsilon, 0] \\
\gamma_{1} R(x+\varepsilon, k), & x \geqslant-\varepsilon
\end{array} .\right.
$$

Here the first line corresponds to the "well", while the second does to the delta-type potential $q(x)=\gamma_{1} \delta(x+\varepsilon)$, for which the first approximation gives an exact solution to the integral equation.

As the first example we apply transformation (2) to delta like potential (8) with two nodes:

$$
q(x)=\gamma_{1} \delta\left(x-x_{1}\right)+\gamma_{2} \delta\left(x-x_{2}\right), \quad x_{21}=x_{2}-x_{1}>0 .
$$


Letting for simplicity $x_{2}=0$, we find that

$$
\psi(x, k)= \begin{cases}\left(1+\frac{\gamma_{1}}{2 k}\right) e^{k x}-\frac{\gamma_{1}}{2 k} e^{2 k x_{1}} e^{-k x}, & x_{1} \leqslant x \leqslant 0 \\ a(k) e^{k x}+b(k) e^{-k x}, & x \geqslant 0\end{cases}
$$

where

$$
a(k)=1+\frac{\gamma_{1}}{2 k}+\frac{\gamma_{2}}{2 k}+\frac{\gamma_{1} \gamma_{2}}{4 k^{2}}\left[1-e^{2 k x_{1}}\right], \quad-b(k)=e^{2 k x_{1}} \frac{\gamma_{1}}{2 k}+\frac{\gamma_{2}}{2 k}+\frac{\gamma_{1}}{2 k} \frac{\gamma_{2}}{2 k}\left(1-e^{2 k x_{1}}\right) .
$$

Thus,

$$
D \log \psi(x, k)=\frac{\psi^{\prime}(x, k)}{\psi(x, k)}=k \frac{\left(1+\frac{\gamma_{1}}{2 k}\right) e^{2 k x}+\frac{\gamma_{1}}{2 k} e^{2 k x_{1}}}{\left(1+\frac{\gamma_{1}}{2 k}\right) e^{2 k x}-\frac{\gamma_{1}}{2 k} e^{2 k x_{1}}}, \quad x_{1}<x<0,
$$

and the jumps of this piece-continuous functions in the nodes of the delta functions, respectively, $\gamma_{1}$ and $\gamma_{2}$ :

$$
\left.\frac{\psi^{\prime}(x, k)}{\psi(x, k)}\right|_{x=x_{1}-0} ^{x=x_{1}+0}=\gamma_{1}, \quad k \frac{a(k)-b(k)}{a(k)+b(k)}=\gamma_{2}+D \log \psi(-0, k) .
$$

The transformed function $\hat{\psi}$ in the formula (2) remains continuous and satisfies the Schrödinger equation (11) with the potential

$$
\hat{q}(x)=-\gamma_{1} \delta\left(x-x_{1}\right)-\gamma_{2} \delta(x)-\left(k_{0}^{2}-\left(\frac{e^{2 k_{0} x}\left(2 k_{0}+\gamma_{1}\right)+e^{2 k_{0} x_{1}} \gamma_{1}}{e^{2 k_{0} x}\left(2 k_{0}+\gamma_{1}\right)-e^{2 k_{0} x_{1}} \gamma_{1}}\right)^{2}\right), \quad x \in\left[x_{1}, 0\right],
$$

for all sufficiently large $k_{0}>0$. However, to keep the transformed potential compactly supported, as $k_{0}$, we should choose the maximal root of $a(k)$ in the right half-plane (5). As $N=2$, the length of the interval $\left[x_{1}, 0\right]$ can be fixed by rescaling $\gamma_{j}$ and letting, for instance, $\gamma_{1}=\gamma_{2}=-1, x_{1}=-1$, we find the only real root $k_{0} \approx 0.63923$ of the function $a(k)$ in halfplane (51). Under such choice of $k_{0}$, potential (22) is compactly supported, but the coefficients $\gamma_{j}$ at the delta functions change sign.

Remark 1. Including delta-type potentials in the theory of Darboux transformations enlarge essentially the potentials of this theory and leads one to an interesting problem on the solutions to the equations

$$
f_{j}^{\prime}+f_{j+1}^{\prime}=f_{j}^{2}-f_{j+1}^{2}+\lambda_{j}-\lambda_{j+1}, \quad j \in \mathbb{Z}
$$

of so-called dressing chain allowing the prescribed jumps $\gamma_{j}$ of first kind at the prescribed points $x_{j}$. The solutions to these equations considered below describe the compositions of the Darboux transformations (21) and corresponds (24) (see below). We recall [5] that smooth periodic in the discrete $j$ solutions to the dressing chain describe finite-gap potentials including the Bargmann potentials.

As it has been already said, while using the zeroes of the second coefficient, to keep the potential real-valued, we use pairs of complex conjugate zeroes and the double Darboux transformation. We consider the superposition of form (2) and the corresponding product of the matrices

$$
F_{j}:=\left(\begin{array}{cc}
f_{j}, & -1 \\
\lambda-\lambda_{j}-f_{j}^{2}, & f_{j}
\end{array}\right), \quad \operatorname{det} F_{j}=\lambda-\lambda_{j} .
$$

In the case $F_{2} F_{1}=\Phi$ we find

$$
\Phi=\left(\begin{array}{cc}
f_{1} g+\lambda_{1}-\lambda & -g \\
\lambda g-\lambda_{1} f_{2}-\lambda_{2} f_{1}-g f_{1} f_{2} & f_{2} g+\lambda_{2}-\lambda
\end{array}\right), \quad g=f_{1}+f_{2} .
$$


It is easy to see that in the general case, the entries $\Phi=\left(\Phi_{i j}\right)$ of an arbitrary product of matrices (2) satisfy the following equations

$$
\begin{gathered}
\frac{d}{d x} \Phi=\left(\begin{array}{cc}
0 & 1 \\
\hat{q}(x)-\lambda & 0
\end{array}\right) \Phi-\Phi\left(\begin{array}{cc}
0 & 1 \\
q(x)-\lambda & 0
\end{array}\right), \quad \Phi=\left[\Phi_{i j}\right], \\
\left\{\begin{array} { l } 
{ \Phi _ { 1 1 } ^ { \prime } = \Phi _ { 2 1 } - ( q ( x ) - \lambda ) \Phi _ { 1 2 } , } \\
{ \Phi _ { 2 2 } ^ { \prime } = ( \hat { q } ( x ) - \lambda ) \Phi _ { 1 2 } - \Phi _ { 2 1 } , }
\end{array} \quad \left\{\begin{array}{l}
\Phi_{12}^{\prime}=\Phi_{22}-\Phi_{11}, \\
\Phi_{21}^{\prime}=\hat{q}(x) \Phi_{11}-q(x) \Phi_{22}+\lambda\left(\Phi_{22}-\Phi_{11}\right) .
\end{array}\right.\right.
\end{gathered}
$$

In the considered case of the double Darboux transform, by these equations we have

$$
\Phi_{12}=-g(x), \quad \Phi_{11}=h(x)-\lambda, \quad \Phi_{22}=-g^{\prime}+\Phi_{11}, \quad \Phi_{21}=h^{\prime}+g(\lambda-q), \quad \hat{q}=q-2 g^{\prime},
$$

and hence,

$$
\Phi_{11} \Phi_{22}=\lambda^{2}-\left(2 h-g^{\prime}\right) \lambda-g^{\prime} h+h^{2}, \quad \Phi_{12} \Phi_{21}=g^{2}(q-\lambda)-g h^{\prime} .
$$

Since the determinant

$$
\operatorname{det} \Phi=\lambda^{2}-2 \alpha \lambda+h^{2}+g h^{\prime}-g^{\prime} h-g^{2} q=\left(\lambda-\lambda_{1}\right)\left(\lambda-\lambda_{2}\right)
$$

is independent of $x$, we finally obtain

$$
\begin{aligned}
& 2 h=g^{2}+g^{\prime}+\lambda_{1}+\lambda_{2}, \quad\left(\frac{h}{g}\right)^{2}+\left(\frac{h}{g}\right)^{\prime}=q(x)+\frac{\lambda_{1} \lambda_{2}}{g^{2}}, \quad f_{1}=\frac{h}{g}-\frac{\lambda_{1}}{g} \\
& g=\frac{\lambda_{2}-\lambda_{1}}{f_{1}-f}, \quad \frac{h}{g}=\frac{f_{1} \lambda_{2}-\lambda_{1} f}{\lambda_{2}-\lambda_{1}}, \quad f^{\prime}+f^{2}=q(x)-\lambda_{2} .
\end{aligned}
$$

It is easy to check that as $\lambda_{2} \neq \lambda_{1}$ the above formulae for $g$ and $g / h$ and the two Ricatti equations

$$
f^{\prime}+f^{2}=q(x)-\lambda_{2}, \quad f_{1}^{\prime}+f_{1}^{2}=q(x)-\lambda_{1}
$$

for the original Schrödginer equation $L \psi=\lambda \psi$ ensures two equations (28) and vice versa. In terms of the transformation operators in work [5] this corresponds to the formulae (see (11), (2)):

$$
\begin{aligned}
& \hat{L} \circ A=A \circ L, \quad A=D^{2}+a_{1} D+a_{2}, \quad A=\left(D-f_{2}\right)\left(D-f_{1}\right), \\
& \left(\hat{q}-D^{2}\right) A=A\left(q-D^{2}\right), \quad A=D^{2}-g D+h-q, \quad A \psi=-g \psi^{\prime}+(h-\lambda) \psi .
\end{aligned}
$$

Returning back to the potential with two nodes and formulae (19), (201), we provide an example of the Darboux transform in the case of two real eigenvalues.

Example 1. As $\gamma_{1}=-1, \gamma_{2}=-1.1, x_{1}=-2$, the graph of $\psi(x, 0)$ has two zeroes. Two real zeroes of the functions a $(k)$ can be found numerically:

$$
a(k)=0, \quad k_{1} \approx 0.665, \quad k_{2} \approx 0.047 .
$$

In Figure 3 we provide the graph of the transformed potential under the single or double Darboux transform.

Let us show that in the case of the degeneration $\left(\lambda_{2}=\lambda_{1}\right)$, the double Darboux transform breaks the property of compact support for the potential.

Lemma 1. Let $\lambda_{2}=\lambda_{1}, \Phi_{12}=-g(x)$ and $\Phi_{11}=h(x)-\lambda$. Then as $q(x)=f_{1}^{\prime}+f_{1}^{2}+\lambda_{1}$, equations (26), (27) are equivalent to the following relations:

$$
g^{\prime}+g^{2}=2 g f_{1}, \quad h=f_{1} g+\lambda_{1}, \quad \hat{q}=q-2 g^{\prime} .
$$

Proof. Indeed, it follows from (28) that

$$
h=f_{1} g+\lambda_{1}, \quad g^{\prime}+g^{2}+\lambda_{2}=2 f_{1} g+\lambda_{1}, \quad f_{1}^{\prime}+f_{1}^{2}+\lambda_{1}=q .
$$

Letting $\lambda_{2}=\lambda_{1}$, we complete the proof. 


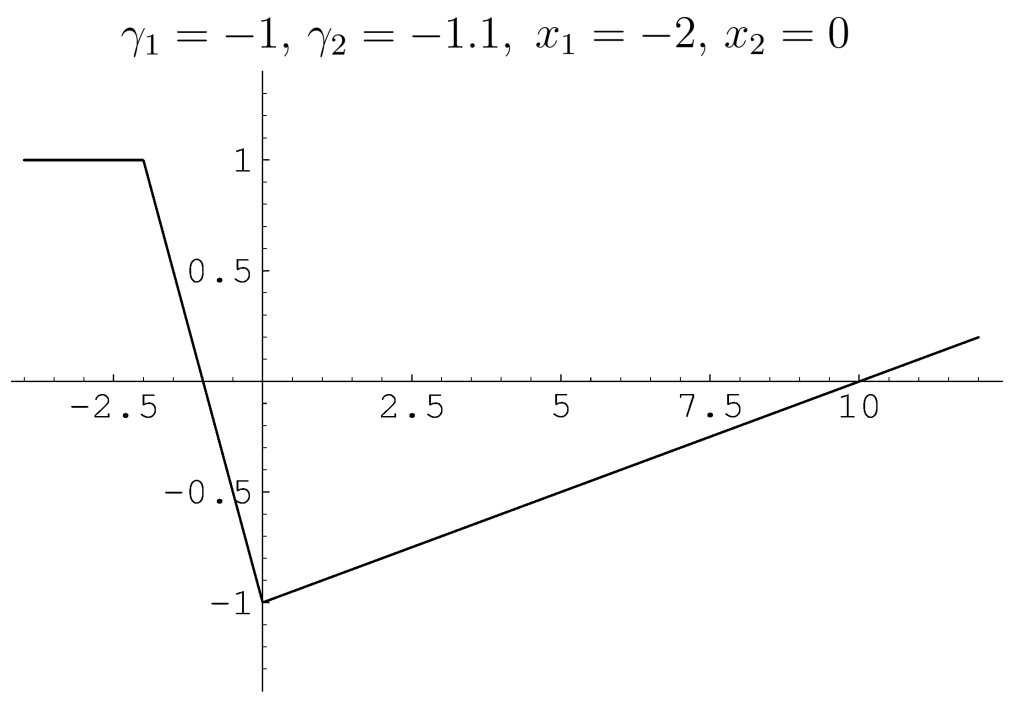

Figure 2. The graph of $\left.\psi(x)\right|_{k=0}$.

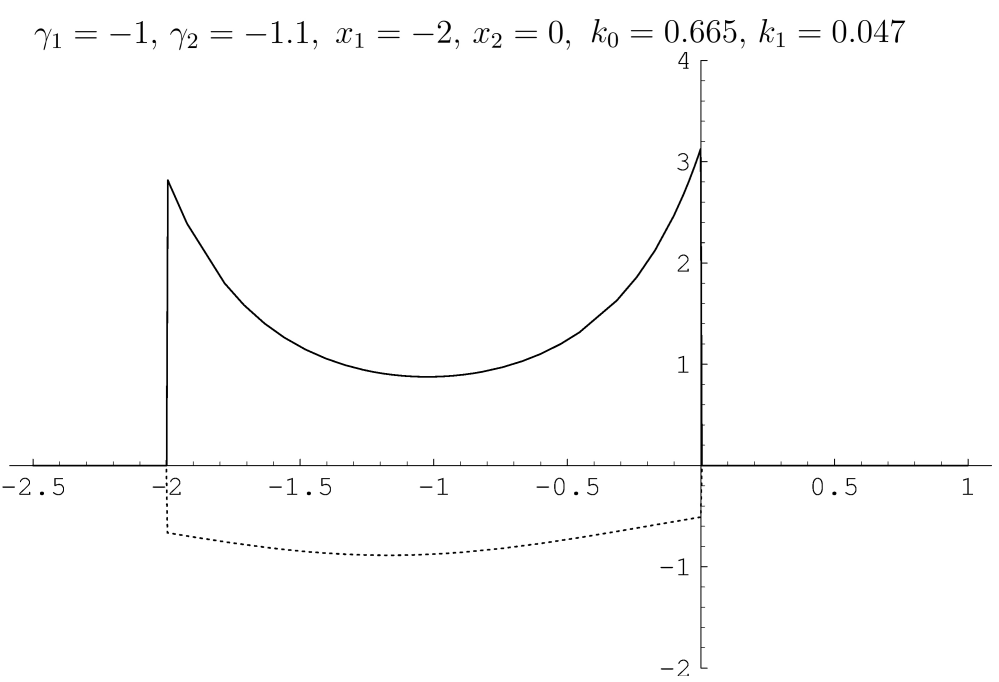

Figure 3. The graph of $\hat{q}(x)+q(x)$ (dotted) and $\hat{q}(x)-q(x)$

In addition to Lemma 1 , we observe that as $\lambda_{2}=\lambda_{1}$, the auxiliary solution $f$ to Ricatti equation (29) can be found by the method of variation of constants:

$$
\begin{aligned}
& f_{1}=\frac{\varphi_{1}^{\prime}}{\varphi_{1}}, \quad f=f_{1}+u \Rightarrow\left(\frac{1}{u}\right)^{\prime}=2 f_{1} \frac{1}{u}+1, \quad u=\frac{z^{\prime}}{z}, \quad z^{\prime}=\frac{1}{\varphi_{1}^{2}} \\
& \varphi=\varphi_{1} z \Rightarrow \varphi^{\prime}=\varphi_{1}^{\prime} z+\frac{1}{\varphi_{1}}, \quad \varphi^{\prime \prime}=\varphi_{1}^{\prime \prime} z=\left(q-\lambda_{1}\right) \varphi .
\end{aligned}
$$

Here $\varphi_{1}$ is an arbitrary solution to the original equation $\varphi_{1}^{\prime \prime}=\left(q-\lambda_{1}\right) \varphi_{1}$ and $\varphi=\varphi_{1} z$ is the found second solution to this equation. As a result of the single transformation (2) with $f=f_{1}$ we find:

$$
\left(D-f_{1}\right) \varphi_{1}=0, \quad\left(D-f_{1}\right) \varphi=\frac{1}{\varphi_{1}}, \quad\left(\frac{1}{\varphi_{1}}\right)^{\prime \prime}=\left(q_{1}-\lambda_{1}\right) \frac{1}{\varphi_{1}}, \quad q_{1}=q-2 f_{1}^{\prime} .
$$


Thus, $v_{1}=-f_{1}$ is a solution to the new Ricatti equation $v^{\prime}+v^{2}=q_{1}-\lambda$ and similar to (32) we find an auxiliary solution $v$ as the sum:

$$
v=-f_{1}+u, \quad u^{\prime}+u^{2}=2 u f_{1} \Rightarrow v+f_{1}=\frac{\varphi_{1}^{2}}{z}, \quad z^{\prime}=\varphi_{1}^{2}
$$

This surely lead us to the formulae in Lemma 1.

Summarizing, we formulate the following statement.

For real-valued compactly supported potentials, the composition of the Darboux transforms corresponding to matrices (24) allow us to get rid of the zeroes of the entire function $2 k a(k)$ located in right half-plane (5) and of finitely many zeroes of the entire function $2 k b(k)$ not lying at the vertical axis. In the second case we apply the double Darboux transform by using the complex conjugate solutions to Ricatti equation (29) and formulae (28).

Remark 2. Under the assumptions of Lemma 1, the functions $f_{j}, j=1,2$, in formulae (24) satisfy the relations

$$
\left(f_{1}+f_{2}\right)^{\prime}=f_{1}^{2}-f_{2}^{2}, \quad f_{2}=\frac{z^{\prime}}{z}-\frac{\varphi_{1}^{\prime}}{\varphi_{1}}, \quad z^{\prime}=\varphi_{1}^{2}, \quad f_{1}=\frac{\varphi_{1}^{\prime}}{\varphi_{1}}
$$

and equations (31) allow us to rewrite the dressing chain in Remark 1 in terms of $z_{j}$

$$
g_{j}=\frac{z_{j}^{\prime}}{z_{j}}, \quad z_{j}^{\prime}=\varphi_{j}^{2}, \quad q_{j+1}=q_{j}-2 g_{j}^{\prime} .
$$

The role of the Ricatti equation is played the known in the spectral theory third order equation with the "Schwarz derivative"

$$
q(x)+k^{2}=\frac{1}{2} \frac{z_{x x x}}{z_{x}}-\frac{1}{4}\left(\frac{z_{x x}}{z_{x}}\right)^{2}, \quad z_{x}=\varphi^{2} .
$$

2.1. Zeroes at the imaginary axis. First let us find out the behavior of the entire function $2 k b(k)$ on the imaginary axis $k=i \xi$ depending on the parameters of delta-type potential (8)). Let $x_{N}=0$ and let the distance between the neighbouring nodes be equal to $\varepsilon$. For the sake of brevity we introduce the auxiliary notations:

$$
\hat{\gamma}_{j}=\frac{\gamma_{j}}{2 k}, \quad e_{j}=e^{2 k x_{j}}, \quad \bar{e}_{j}=e^{-2 k x_{j}}, \quad e=e_{j} \bar{e}_{j+1}=e^{-2 k \varepsilon} .
$$

Multiplying the scattering matrices of delta-type potentials (see [2]), we find, for instance, that as $N=3$,

$$
\begin{gathered}
-b(k)=\hat{\gamma}_{3}\left(1+\hat{\gamma}_{1}\right)\left(1+\hat{\gamma}_{2}\right)+e^{2} \hat{\gamma}_{1}\left(1-\hat{\gamma}_{2}\right)\left(1-\hat{\gamma}_{3}\right)+e \hat{\gamma}_{2}\left(1+\hat{\gamma}_{1}\right)\left(1-\hat{\gamma}_{3}\right)-e \hat{\gamma}_{1} \hat{\gamma}_{2} \hat{\gamma}_{3}, \\
e_{1} b(-k)=\hat{\gamma}_{1}\left(1+\hat{\gamma}_{2}\right)\left(1+\hat{\gamma}_{3}\right)+e^{2} \hat{\gamma}_{3}\left(1-\hat{\gamma}_{1}\right)\left(1-\hat{\gamma}_{2}\right)+e \hat{\gamma}_{2}\left(1-\hat{\gamma}_{1}\right)\left(1+\hat{\gamma}_{3}\right)-e \hat{\gamma}_{1} \hat{\gamma}_{2} \hat{\gamma}_{3} .
\end{gathered}
$$

Since $\bar{b}(k)=b(-k)$ on the real axis, the condition $\gamma_{1}=\gamma_{3}$ ensures that $b(i \xi)=0 \Leftrightarrow \bar{b}(i \xi)=0$ and $e_{1} b(-k)=-\bar{e}_{N} b(k)$. It is obvious (see (13)) that the latter condition is satisfied for all even up to a shift delta-type potentials (8) and that for such potentials the problem on the zeroes of the entire function $2 k b(k)$ on the imaginary axis is reduced to the zeroes of trigonometrical quasi-polynomials with real coefficients. The problem on the structure of zero set for such polynomials was made numerically in work [3]. In the following example these zeroes form an integer lattice up to the choice of $\varepsilon$ in formula (34).

Example 2. As $N=2$, thanks to (20), the roots of $b(k)$ are determined by following simple equation

$$
e^{2 k x_{1}}=\frac{1+2 k / \gamma_{1}}{1-2 k / \gamma_{2}} \Rightarrow e^{2 k x_{1}}=1, \quad \text { if } \gamma_{1}+\gamma_{2}=0
$$




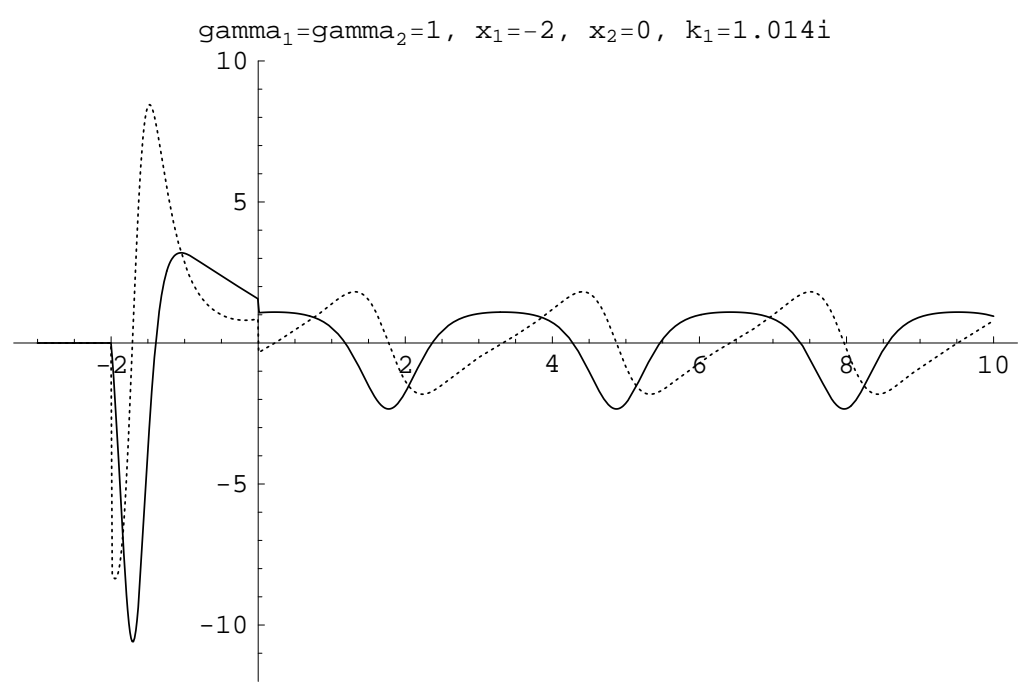

Figure 4. Graphs for $\operatorname{Re} \hat{q}(x) \operatorname{Im} \hat{q}(x)$ (dotted) for an imaginary zero of $b(k)$

As $\gamma_{1}+\gamma_{2}=0$, the roots of the equation $e^{2 k x_{1}}=1$ form a regular lattice on the imaginary axis and

$$
\frac{b(k)}{a(k)}=\left(1+\beta_{1} k\right) \frac{\exp 2 k x_{1}-1}{\beta_{1}^{2} k^{2}+\exp 2 k x_{1}-1}, \quad \beta_{1}=\frac{2}{\gamma_{1}}, \quad k=i \xi .
$$

The Fourier transform of this function on the imaginary axis and the corresponding solution to integral equation (17) (cf. [4]) seems to lead a delta-type potential with two nodes at the points $x_{1}$ and $x_{2}=0$.

As it was mentioned in the end of Section 1, the Darboux transforms related with pair (15) of zeroes on the imaginary axis of the entire function $2 k b(k)$ lead out the class of the real compactly supported potentials and produce potentials with supported on the half-line similar to one shown on Figure 4. This graph shows the application of Lemma 1 in considered case (35). The form of the transformed potential $\hat{q}=q-2 g^{\prime}$ is determined by means of the function $g(x)$ introduced as the solution to the Cauchy problem for the system of first order differential equations (see Lemma 1):

$$
g^{\prime}+g^{2}=2 f_{1} g, \quad f_{1}^{\prime}+f_{1}^{2}+\lambda_{1}=q, \quad g\left(x_{1}\right)=2 k_{1}, f_{1}\left(x_{1}\right)=k_{1}, \quad \lambda_{1}=-k_{1}^{2} .
$$

To specify the details we involve the function

$$
\varphi_{1}(x)= \begin{cases}e^{k x}+\frac{\gamma_{1}}{k} \sinh (k x), & x_{1} \leqslant x \leqslant 0 \\ a(k) e^{k x}, \quad x>0, & k \equiv k_{1}, \quad b\left(k_{1}\right)=0 .\end{cases}
$$

It is easy to see that $\hat{q}(x)=0$ as $x<x_{1}$ and for $x>x_{1}$ all can be expressed in terms of this function $\varphi_{1}$ (see Remark 2).

2.2. Acknowledgments. We thank V.E. Adler and R.Ch. Kulaev for an interest to the work and useful remarks.

\section{BIBLIOGRAPHY}

1. A.B. Shabat. Inverse spectral problem for delta potentials // Pisma ZhETF. 102:9, 705-708 (2015). [JETP Lett. 102:9, 620-623 (2015).]

2. A.B. Shabat. Scattering theory for delta-type potentials // Teor. Matem. Fiz. 183:1, 105-119 (2015). [Theor. Math. Phys. 183:1, 540-552 (2015).]

3. M.Sh. Badakhova, O.Y. Veremeenko, A.B. Shabat. Asymptotic behavior of generalized eigenvalues of the Schrödinger operator // Vladikavkaz. Matem. Zhurn. 16:4, 34-40 (2014). (in Russian). 
4. E. Korotyaev. Inverse scattering on the real line // Inverse Problems. 21:1, 325-341 (2005).

5. A. P. Veselov, A.B. Shabat. Dressing chains and spectral theory of the Schrödinger operator // Funkts. Anal. Pril. 27:2, 1-21 (1993).

6. V.Bargmann. Remarks on the determination of a central field of force from the elastic scattering phase shifts // Phys. Rev. 75:2, 301-303 (1949).

Mukhtar Shamil'evich Badakhov,

Karachaevo-Cherkess State University named after U.D. Aliev,

Lenin str. 23,

369200, Karachaevsk, Russia

Institute of Mathematics, Ufa Scientific Center, RAS

Chernyshevsky str. 112,

450008, Ufa, Russia

E-mail: badahov92@mail.ru

Alexei Borisovich Shabat,

Landau Institute for Theoretical Physics,

Akademika Semenova av. 1-A,

142432, Chernogolovka, Russia

Karachaevo-Cherkess State University named after U.D. Aliev,

Lenin str. 23,

369200, Karachaevsk, Russia

Institute of Mathematics, Ufa Scientific Center, RAS

Chernyshevsky str. 112,

450008, Ufa, Russia

E-mail: shabatab@mail.ru 